\title{
Improving EPMA Analyses Using Mean Atomic Number Backgrounds
}

\author{
D.A Wark* and J.J. Donovan** \\ * GE Global Research Center, One Research Circle, Niskayuna, NY 12309 \\ **CAMCOR, University of Oregon, Eugene, OR 97403-1241
}

Faced with time constraints and with beam sensitive materials, electron probe micro-analyser (EPMA) operators often desire faster analyses to save time and/or to minimize sample damage from exposure to the electron beam. One of the most common approaches to decreasing analytical time is to reduce the amount of time spent counting x-rays both on peak and on background. This results in fewer x-ray counts, however, which translates into reduced analytical precision and higher detection limits. Increasing the beam current can compensate for this reduced count time, but may also result in increased sample damage and an attendant decrease in accuracy.

Here we assess the benefits of an alternative approach to speeding up analyses - the Mean Atomic Number (MAN) background correction [I]. Instead of measuring background count rates using typical off-peak methods, the analyst substitutes a value for background that is based on an empirical fit to the (near) linear relation between bremsstrahlung count rate and mean atomic number of the sample as measured in materials not containing the element of interest. By eliminating off-peak background measurements, analyses are faster (commonly by $>50 \%$ ). Additionally, spectrometer motion is decreased, thereby improving reproducibility and reducing instrument wear. Accuracy of the technique, however, has not to our knowledge been rigorously evaluated.

With the goal of evaluating the accuracy of MAN analyses across a broad range of element concentrations and spectrometer configurations, we measured 11 elements (concentrations ranging $<0.01$ to $60 \mathrm{wt} \%$ ) on four spectrometers (using TAP, PET, and LIF crystals) at 300 locations on metal alloys of varying composition. We first used the conventional off-peak (background interpolation) approach, using Probe for EPMA software on a JEOL 8200 instrument. These "benchmark" (BM) data were then reprocessed after substituting MAN background values based on fits to background counts on pure metal standards. Next, we divided the difference between each of the $>3300 \mathrm{MAN}$ and $\mathrm{BM} w \mathrm{wt} \%$ values $\left(\mathrm{C}_{\mathrm{MAN}}-\mathrm{C}_{\mathrm{BM}}\right)$ by their $1 \sigma$ analytical uncertainties, reasoning that "acceptable" differences are those that are analytically indistinguishable. Recognizing that peak/background ratios decrease and therefore the influence of background inaccuracies increase at lower element concentrations, these values were then plotted against the benchmark element concentrations (Figure 1).

Confirming the accuracy of MAN backgrounds, over $98 \%$ of the $\mathrm{C}_{\mathrm{MAN}}$ values fall within $1 \sigma$ of benchmark values for all concentrations above $4 \mathrm{wt} \%$. This number drops to $80 \%$ for all data above $0.2 \mathrm{wt} \%$, but it is worth noting that only a few MAN values - most corresponding to concentrations near detection limit - fall outside the $\pm 3 \sigma$ bounds. Even these differences are acceptable for most purposes: for example, at $3 \mathrm{wt} \%$, a $3 \sigma$ difference indicates a MAN value different from the benchmark by only $0.1 \mathrm{wt} \%$. Worth consideration, too, is the possibility that MAN values are more accurate than our "benchmark" values: This turned out to be the case for one element (due to a misplaced off-peak background setting) when this study was first performed. Indeed, where 
measurement of conventional off-peak backgrounds is compromised by interferences from secondary elements or by continuum artifacts such as detector or sample absorption edges, the MAN background may be the only choice.

This study demonstrates that MAN background calculations can be at least as accurate as conventional background measurements for element concentrations down to a few tenths of a percent, with significant time savings. In our study, we reduced analysis time by $40 \%$, but greater savings can be expected if only a single element is analyzed per spectrometer because spectrometer drive time is not reduced using MAN. Either way, adoption of the MAN approach allows the operator to (a) reduce instrument time, (b) obtain a larger number of analyses, and/or (c) increase the precision of every analysis by increasing the time spent measuring on peak.

References

[1] J.J. Donovan and T.N. Tingle, Jour. Microscopy. 2 (1996) 1.

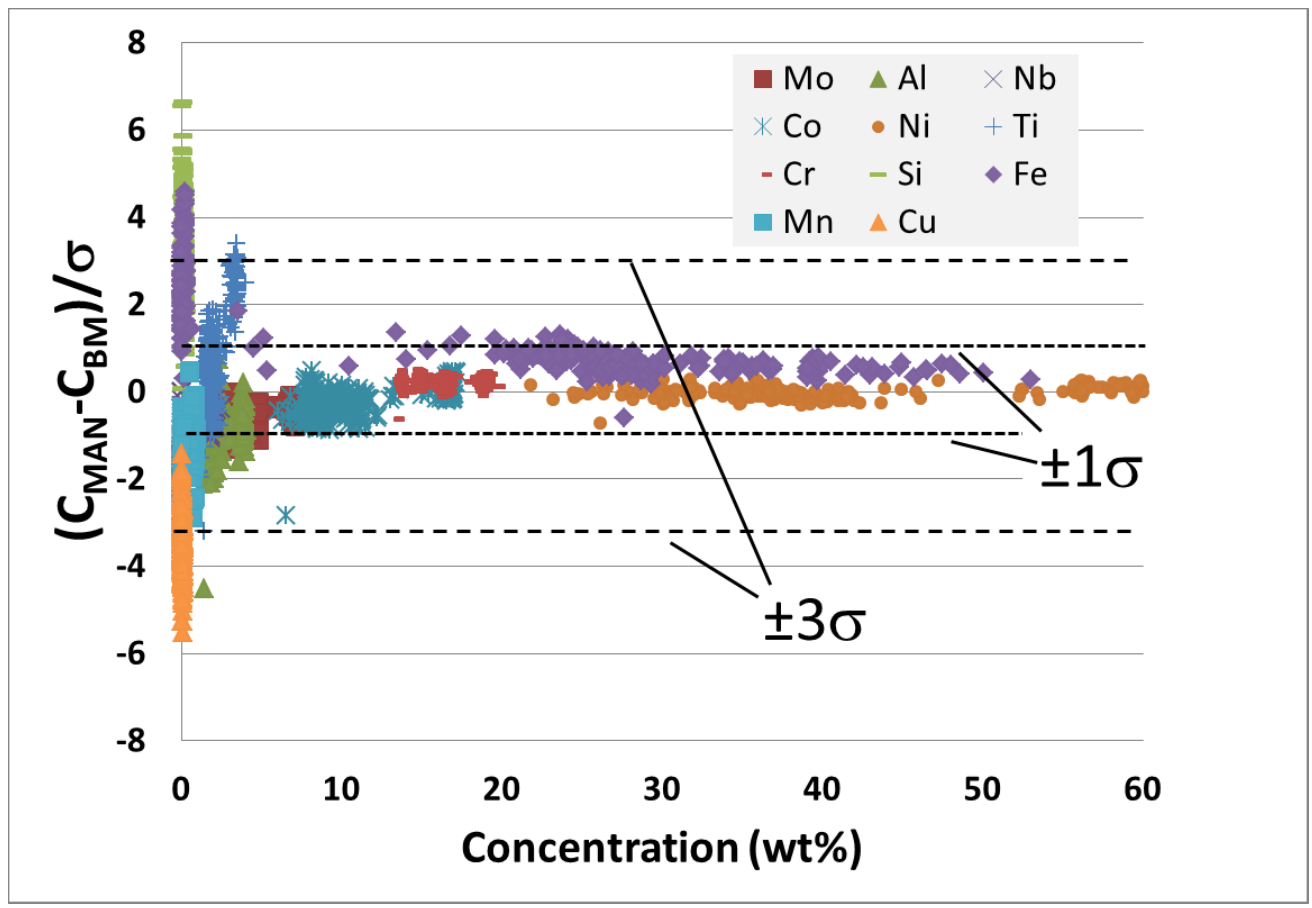

FIG. 1. Plot illustrating difference between element concentrations determined by MAN and benchmark (BM) off-peak methods, divided by analytical uncertainty (see text) for $>3300$ individual analyses, as a function of element concentration. Dashed lines encompass data for which the values for each method differ by $\pm 1 \sigma$ and $3 \sigma$, as indicated. 